7th International Workshop on Astronomy and

Relativistic Astrophysics (IWARA 2016)

International Journal of Modern Physics: Conference Series

Vol. 45 (2017) 1760026 (8 pages)

(C) The Author(s)

DOI: $10.1142 / \mathrm{S} 2010194517600266$

\title{
Hybrid Stars in the Framework of NJL Models
}

\author{
Gustavo A. Contrera \\ Consejo Nacional de Investigaciones Científicas y Técnicas (CONICET) \\ Godoy Cruz 2290, 1425 Buenos Aires, Argentina \\ Facultad de Ciencias Astronómicas y Geofísicas \\ Universidad Nacional de La Plata (UNLP) \\ Paseo del Bosque S/N (1900), La Plata, Argentina \\ Facultad de Ciencias Exactas \\ Universidad Nacional de La Plata (UNLP) \\ calle 49 y 115, La Plata, Argentina \\ contrera@fisica.unlp.edu.ar \\ Milva Orsaria \\ Consejo Nacional de Investigaciones Científicas y Técnicas (CONICET) \\ Godoy Cruz 2290, 1425 Buenos Aires, Argentina \\ Facultad de Ciencias Astronómicas y Geofísicas \\ Universidad Nacional de La Plata (UNLP) \\ Paseo del Bosque S/N (1900), La Plata, Argentina \\ Department of Physics, San Diego State University (SDU) \\ 5500 Campanile Drive, 92182 San Diego, CA, USA \\ morsaria@fcaglp.unlp.edu.ar \\ I. F. Ranea-Sandoval \\ Consejo Nacional de Investigaciones Científicas y Técnicas (CONICET) \\ Godoy Cruz 2290, 1425 Buenos Aires, Argentina \\ Facultad de Ciencias Astronómicas y Geofísicas \\ Universidad Nacional de La Plata (UNLP) \\ Paseo del Bosque $S / N$ (1900), La Plata, Argentina \\ iranea@fcaglp.unlp.edu.ar \\ Fridolin Weber \\ Department of Physics, San Diego State University (SDU) \\ 5500 Campanile Drive, 92182 San Diego, CA, USA \\ Center for Astrophysics and Space Sciences \\ University of California \\ 92093 San Diego, La Jolla, CA, USA \\ fweber@mail.sdsu.edu \\ Published 15 August 2017
}

This is an Open Access article published by World Scientific Publishing Company. It is distributed under the terms of the Creative Commons Attribution 4.0 (CC-BY) License. Further distribution of this work is permitted, provided the original work is properly cited. 


\title{
G. A. Contrera et al.
}

\begin{abstract}
We compute models for the equation of state (EoS) of the matter in the cores of hybrid stars. Hadronic matter is treated in the non-linear relativistic mean-field approximation, and quark matter is modeled by three-flavor local and non-local Nambu-Jona-Lasinio (NJL) models with repulsive vector interactions. The transition from hadronic to quark matter is constructed by considering either a soft phase transition (Gibbs construction) or a sharp phase transition (Maxwell construction). We find that high-mass neutron stars with masses up to $2.1-2.4 M_{\odot}$ may contain a mixed phase with hadrons and quarks in their cores, if global charge conservation is imposed via the Gibbs conditions. However, if the Maxwell conditions is considered, the appearance of a pure quark matter core either destabilizes the star immediately (commonly for non-local NJL models) or leads to a very short hybrid star branch in the mass-radius relation (generally for local NJL models).
\end{abstract}

Keywords: Hybrid Stars, Neutron Stars, Dense Matter, Phase Transitions.

PACS numbers:26.60.-c, 26.60.Kp, 25.75.Nq, 97.60.Jd

\section{Introduction}

The question of whether or not quark matter exists in neutron stars has received renewed interest (see Refs. ${ }^{1-3}$ and references therein) by the discovery of the two massive neutron stars (NS) J1614-2230 $\left(1.97 \pm 0.04 M_{\odot}\right.$ (see Ref. $\left.{ }^{4}\right)$, recently updated to $1.928 \pm 0.017 M_{\odot}$ (see Ref. $\left.{ }^{5}\right)$ and J0348+0432 $\left(2.01 \pm 0.04 M_{\odot}\right.$ (see Ref. $\left.{ }^{6}\right)$ ). Aside from these two massive objects, there may exist even heavier neutron stars which are known as "Black Widow Pulsars", such as B1957+20 $\left(2.39_{-0.29}^{+0.36} M_{\odot}\left(\right.\right.$ see Ref. $\left.\left.{ }^{7}\right)\right)$. If the dense interior of a NS is indeed converted to quark matter, ${ }^{8-10}$ it must be threeflavor quark matter since it has lower energy than two-flavor quark matter. And just as for the hyperon content of NS, strangeness is not conserved on macroscopic time scales, which allows them to convert confined hadronic matter to three-flavor quark matter until equilibrium brings this process to a halt. As first realized by Glendenning, ${ }^{8}$ the presence of quark matter in these stars enables the hadronic regions of the mixed phase to become more isospin symmetric than in the pure phase by transferring electric charge to the quark phase. The symmetry energy can be lowered thereby at only a small cost in rearranging the quark Fermi surfaces. The stellar implication of this charge rearrangement is that the mixed phase region of a NS will have positively charged regions of nuclear matter and negatively charged regions of quark matter. ${ }^{8}$ This should have important implications for its electric and thermal properties.

It has been shown ${ }^{11-13}$ that the appearance of a mixed phase of quarks and hadrons in NS is favored when the value of the surface tension between nuclear matter and quark matter is lower than $\sigma \sim 40 \mathrm{MeV} / \mathrm{fm}^{2}$. Some recent studies ${ }^{14-19}$ predict the value of the surface tension around $\sigma \sim 5-30 \mathrm{MeV} / \mathrm{fm}^{2}$, while oth$\operatorname{ers}^{11-13,20,21}$ obtain a value around $\sigma \sim 50-300 \mathrm{MeV} / \mathrm{fm}^{2}$. Comparing those works it can be seen that the results are strongly model dependent. Thus, the appearance of a mixed quark-hadron phase in NS is therefore an open issue.

Our study is based on NS containing deconfined quark matter, i.e. quark-hybrid stars (QHSs). As in previous works, ${ }^{1,2,22-24}$ we use three-flavor local and non-local 
Nambu-Jona-Lasinio (NJL) models with repulsive vector interactions to describe the quark matter phase. To model hadronic matter, we adopt the non-linear Walecka model using different nuclear parametrizations adjusted to the properties of infinite nuclear matter at saturation density. A phase transition between hadronic matter and quark matter is constructed via the Gibbs and Maxwell conditions, depending on the assumption of the surface tension at the hadron-quark interface.

\section{Hadron-Quark Phase Transition}

Several theoretical works ${ }^{25-28}$ have shown that a first-order phase transition between hadronic and quark matter ought to happen for cold and dense hadronic matter as existing in the cores of NS. The density at which such a phase transition occurs is not well defined, but it is supposed to be several times the nuclear saturation density. To model this phase transition, two different treatments are generally adopted. These are A) the Maxwell construction, in which a sharp phase transition between hadronic and quark matter takes place, which rules out the existence of a mixed phase; B) the Gibbs construction, where pressure in the mixed phase varies, which gives rise to the appearance of a mixed phase.

The value of the surface tension at the interface separating hadrons from quarks is crucial since it determines which scenario ought to be used to study the phase transition. Theoretical studies ${ }^{11-13}$ suggest that if the surface tension between hadronic and quark matter is around $5-40 \mathrm{MeV} / \mathrm{fm}^{2}$, the Gibbs construction would be favored. Otherwise the transition should be described by the Maxwell construction. Given the uncertainties in the value of the surface tension, both scenarios should be considered plausible.

For example, the study of the constant speed of sound (CSS) parametrization for quark matter ${ }^{29}$ assumes that the Maxwell construction is the proper one to describe the phase transition, which is determined by the crossing point between the hadronic and quark matter equation of state (Fig. 2). This phase transition is isobaric and occurs over a finite density range defined by

$$
P^{H}\left(\mu_{B}^{H}, \mu_{e}^{H}\right)=P^{q}\left(\mu^{q}, \mu_{e}^{q}\right), \quad \mu_{B}^{H}=3 \mu^{q},
$$

where $\mu_{B}^{H}$ and $\mu^{q}$ are the hadronic and quark chemical potentials, respectively. For the Maxwell transition, the baryonic chemical potential is continuous while the electron chemical potential, $\mu_{e}$, jumps at the interface between the hadronic and quark phases. The phase transition is abrupt and the pressure within the transition region is constant. As a consequence, the mixed phase region of the Maxwell transition is strictly excluded from the cores of neutron stars, leading to a density discontinuity at the interface between confined hadronic matter and deconfined quark matter. The situation is drastically different for the Gibbs case, as illustrated in Fig. 1, where extended regions of mixed quark-hadron matter in the cores of neutron stars can be obtained, $\mu_{e}$ is continuous, and the pressure varies monotonically with density (see Fig. 2). Electric charge neutrality is fulfilled locally for the Maxwell construction, 

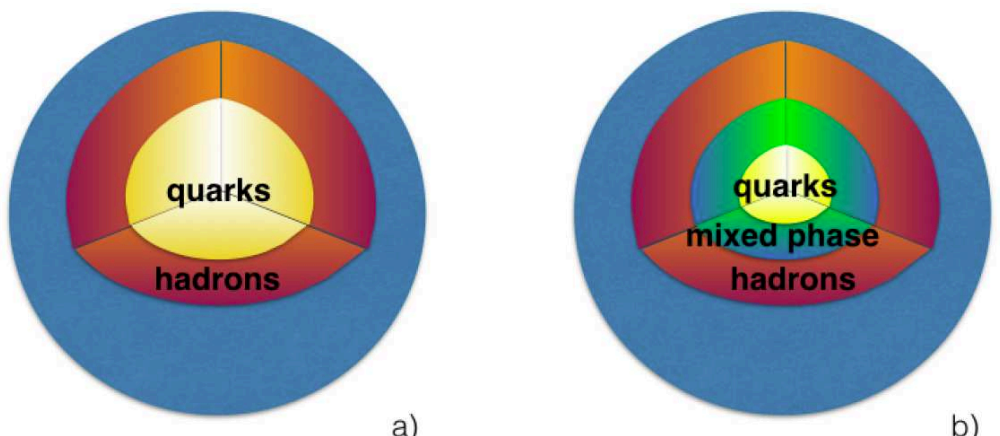

a)

b)

Fig. 1. (Color online) Schematic illustration of the interior structure of a hybrid star for the Maxwell construction (a) and the Gibbs construction (b). Regions of quark-hadron matter are only obtained for the latter.

in contrast to the Gibbs case where charge neutrality is imposed as a global constraints. The condition for phase equilibrium between hadronic and quark matter for the Gibbs construction is given by

$$
P^{H}\left(\mu_{B}^{H}, \mu_{e}^{H}\right)=P^{q}\left(\mu^{q}, \mu_{e}^{q}\right), \quad \mu_{B}^{H}=3 \mu_{B}^{q}, \quad \mu_{e}^{H}=\mu_{e}^{q},
$$

with the baryon and electron chemical potentials continuous at the phase boundary.

For the Maxwell construction, the necessary and sufficient condition for the phase transition to occur can be readily seen in the pressure versus baryon chemical potential diagram. Namely, if there is a point at which the equations of state of the two phases intersect, then a phase transition occurs. This is shown in the right panel of Fig. 2.

For the Gibbs construction, the crossing of the quark and hadronic EoS is a necessary condition, but it is not a sufficient one. This is so because the Gibbs condition is satisfied by imposing global charge conservation so that pressure, energy density, and baryon and electron chemical potentials vary monotonically as the phase transition proceeds. For this reason it is convenient to include a new parameter which takes into account the volume proportion of quark matter and the variation of the thermodynamic quantities point by point in the mixed phase (see Refs. 1, 2 for details).

Since there is no sharp phase transition for the Gibbs construction between hadronic and quark matter but rather a mixed phase, it is not possible to perform the CSS parametrization for the quark matter EoS, as proposed in Ref. 22. We also note that, for the Gibbs treatment, stellar cores made of pure quark matter are not obtained for the different EoS considered in this work. However, a mixed phase of quarks and hadrons is always present, consisting of $\sim 30-60 \%$ of quark matter for the local NJL parametrization, and $\sim 30-35 \%$ for the non-local NJL models, depending on the combination of the hadronic and quark matter parameters. 

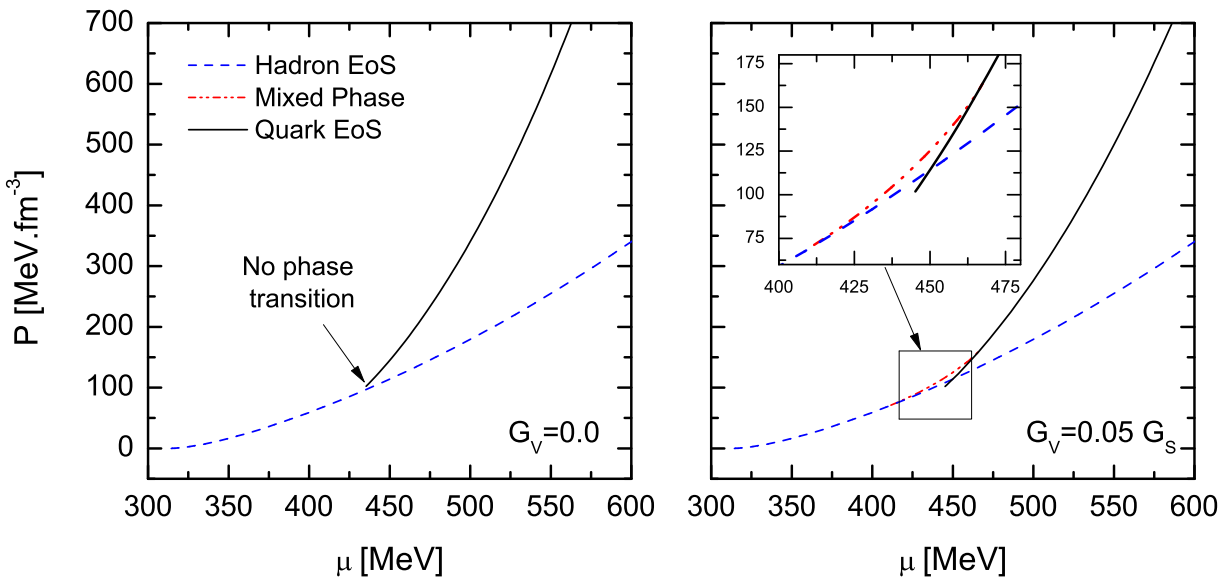

Fig. 2. (Color online) Pressure, $P$, as a function of quark chemical potential, $\mu_{q}$. In the left panel (without vector interactions, $G_{V}=0.0$ ), there is no hadron-quark phase transition since the hadronic and quark matter EoS do not cross. In the panel on the right (with vector interactions, $G_{V}=0.05 G_{S}$ ), the crossing of both EoS determines the density at which the hadron-quark phase transition occurs for the Maxwell construction. The insert shows the smooth mixed-phase region characteristic of the Gibbs transition. These plots correspond to the NL3 parametrization $\left(\chi_{\sigma}=0.7\right)$ for the hadron EoS and non-local NJL (Set II in Ref. 22) for the quark EoS.
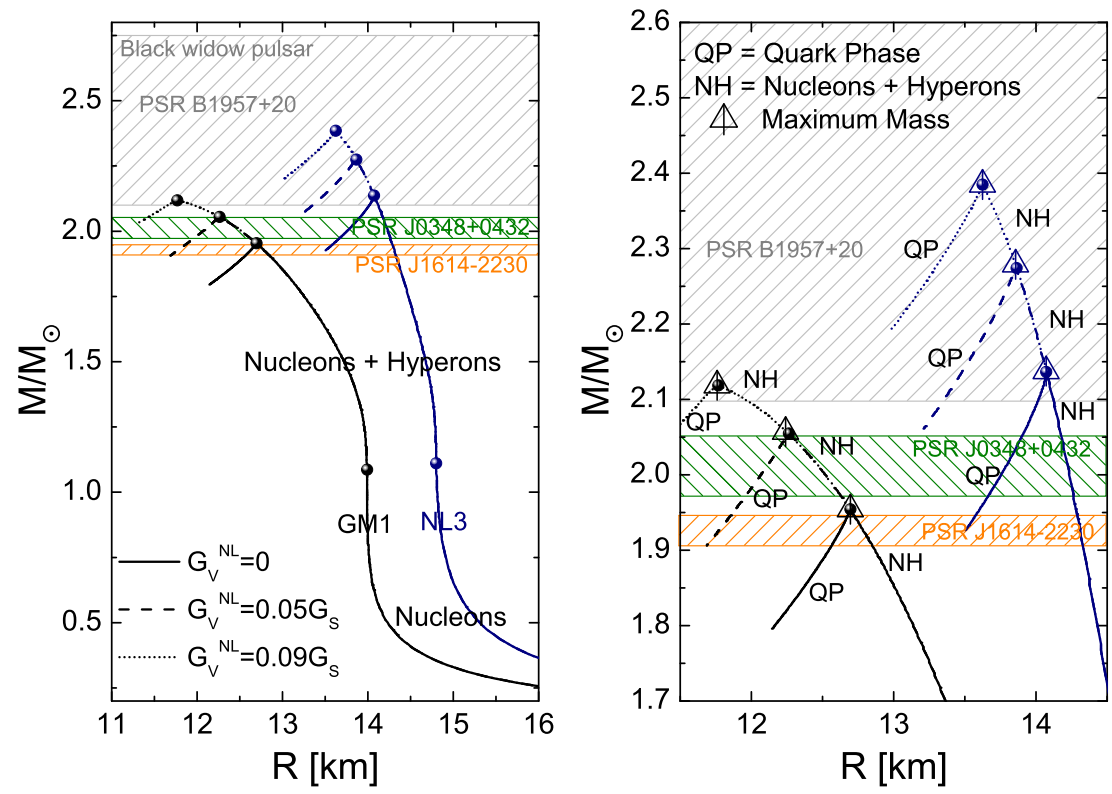

Fig. 3. (Color online) Mass-radius relationship of quark-hybrid stars for the Maxwell construction, computed for universal coupling of hyperons and nuclear parametrizations GM1 and NL3. 

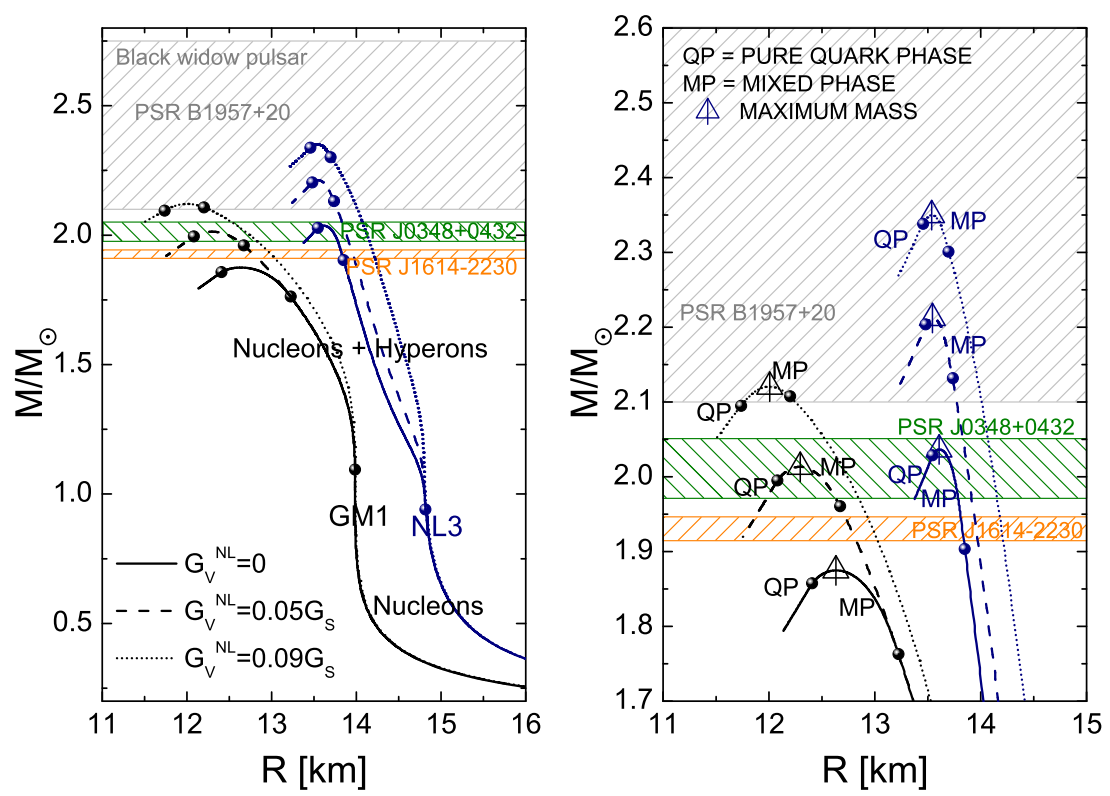

Fig. 4. (Color online) Same as Fig. 3 but for the Gibbs construction.

\section{Results and Conclusions}

The indication of this work is that if the Gibbs condition is used to model phase equilibrium between hadronic and quark matter, high-mass neutron stars, such as the pulsars J3048+0432, J1614-2230 and B1957+20, may contain significant amounts of quark-hadron matter in their cores. The established values are $30-35 \%$ of quark matter in case of a non-local NJL model, and $\sim 30-60 \%$ for the local NJL model, provided the surface tension between hadronic and quark matter is low, as suggested in the recent literature (Refs. ${ }^{14,16-19}$ )

For the non-local NJL model and the NL3 parametrization for the hadronic phase, we find that pure quark matter would not exist in stable neutron stars, since only neutron stars that lie on the left of the mass peak have dense enough cores to contain quark matter. Such stars, however, are unstable against radial oscillations and thus could not exist stably in the universe. With increasing stellar mass (that is, density) the stellar cores are composed of either nucleons, nucleons and hyperons, or a mixed phase of nucleons, hyperons, and quark matter (see Fig. 4).

On the other hand, the main conclusion which follows from using the Maxwell construction is that the non-local NJL models that we have studied generally do not lead to hybrid stars, while the local NJL models do for some parametrizations. These stars, however, cover only a very small range of masses and radii. This is different when the Gibbs condition is used to determine the phase transition. Most of the non-local NJL EoSs lack hybrid branches because the jump in energy density 
at the phase transition is so large that the quark matter core destabilizes the star immediately (see Fig. 3).

In the future, it would be interesting to study the impact of color superconductivity in the quark phase, and different hadronic lagrangians (e.g., density dependent nuclear field theory) on the bulk properties of neutron stars.

\section{Acknowledgments}

G. A. C., M. O. and I. F. R-S thank CONICET and UNLP (Argentina) for financial support. M. O. thanks the American Physical Society for an International Research Travel Award. is supported by the National Science Foundation (USA) under Grant PHY-1411708.

\section{References}

1. M. Orsaria, H. Rodrigues, F. Weber, and G. A. Contrera, Phys. Rev. D 87, 023001 (2013).

2. M. Orsaria, H. Rodrigues, F. Weber, and G. A. Contrera, Phys. Rev. C 89, 015806 (2014).

3. F. Weber, G. A. Contrera, M. G. Orsaria, W. Spinella, and O. Zubairi, Mod. Phys. Lett. A 29, 1430022 (2014).

4. P. Demorest, T. Pennucci, S. Ransom, M. Roberts, and J. Hessels, Nature 467, 1081 (2010).

5. E. Fonseca et al., Astrophys. J. 832, 167 (2016).

6. W. Lynch et al. Astrophys. J 763, 81 (2013); J. Antoniadis et al., Science 340, 6131 (2013).

7. M. H. van Kerkwijk, R. Breton, and S. R. Kulkarni, Astrophys. J. 728, 95 (2011).

8. N. K. Glendenning, Compact Stars, Nuclear Physics, Particle Physics, and General Relativity, 2nd ed. (Springer-Verlag, New York, 2000); N. K. Glendenning, Phys. Rev. D 46, 1274 (1992); N. K. Glendenning, Phys. Rep. 342, 393 (2001).

9. F. Weber, Pulsars as Astrophysical Laboratories for Nuclear and Particle Physics, High Energy Physics, Cosmology and Gravitation Series (IOP Publishing, Bristol, Great Britain, 1999).

10. F. Weber, Prog. Part. Nucl. Phys. 54, 193 (2005).

11. M. G. Alford, K. Rajagopal, S. Reddy, and F. Wilczek, Phys. Rev. D 64, 074017 (2001).

12. D. N. Voskresensky, M. Yasuhira, and T. Tatsumi, Nucl. Phys. A 723, 291 (2003).

13. T. Tatsumi, M. Yasuhira, and D. N. Voskresensky, Nucl. Phys. A 718, 359 (2003).

14. L. F. Palhares and E. S. Fraga, Phys. Rev. D 82, 125018 (2010).

15. T. Endo, Phys. Rev. C 83, 068801 (2011).

16. M. B. Pinto, V. Koch, and J. Randrup, Phys. Rev. C 86, 025203 (2012).

17. W. y. Ke and Y. x. Liu, Phys. Rev. D 89, 074041 (2014).

18. B. W. Mintz, R. Stiele, R. O. Ramos, and J. Schaffner-Bielich, Phys. Rev. D 87, 036004 (2013).

19. N. Yasutake, R. Lastowiecki, S. Benic, D. Blaschke, T. Maruyama, and T. Tatsumi, Phys. Rev. C 89, 065803 (2014).

20. T. A. S. d. Carmo, G. Lugones, and A. G. Grunfeld, J. Phys. G 40, 035201 (2013).

21. G. Lugones, A. G. Grunfeld, and M. Al Ajmi, Phys. Rev. C 88, 045803 (2013). 
22. I. F. Ranea-Sandoval, S. Han, M. G. Orsaria, G. A. Contrera, F. Weber, and M. G. Alford, Phys. Rev. C 93, 045812 (2016).

23. W. M. Spinella, F. Weber, G. A. Contrera, and M. G. Orsaria, Eur. Phys. J. A 52, $61(2016)$

24. S. M. de Carvalho, R. Negreiros, M. Orsaria, G. A. Contrera, F. Weber, and W. Spinella, Phys. Rev. C 92, 035810 (2015).

25. S. Benic, D. Blaschke, D. E. Alvarez-Castillo, T. Fischer, and S. Typel, Astron. Astrophys. 577, A40 (2015).

26. M. Buballa, F. Neumann, M. Oertel, and I. Shovkovy, Phys. Lett. B 595, 36 (2004).

27. R. Negreiros, V. A. Dexheimer, and S. Schramm, Phys. Rev. C 85, 035805 (2012).

28. A. Kurkela, P. Romatschke, and A. Vuorinen, Phys. Rev. D 81, 105021 (2010).

29. M. G. Alford, S. Han, and M. Prakash, Phys. Rev. D 88, 083013 (2013). 